\title{
La prevención e intervención en el cyberbullying: ¿qué papel juegan las
}

\author{
familias?
}

\section{Prevention and intervention in cyberbullying: what role do families play?}

\author{
Diana Priegue Caamaño, Leticia López Castro, Paula Outón Oviedo \\ Universidade de Santiago de Compostela
}

\begin{abstract}
Resumen
La prevención del cyberbullying constituye uno de los mayores desafíos que deberemos afrontar los investigadores sociales en los próximos años. Justamente por ello, analizamos la relevancia que los expertos han otorgado a las variables familiares en la prevalencia del cyberbullying, llevando a cabo una revisión bibliográfica de las investigaciones realizadas al respecto. Entre las conclusiones obtenidas destaca la necesidad de explorar en mayor medida la importancia del contexto familiar por ser una variable clave de cara la puesta en marcha de programas socioeducativos centrados en la prevención y reducción del cyberbullying.

Palabras clave: cyberbullying, prevención, intervención, familia.
\end{abstract}

\begin{abstract}
The prevention of cyberbullying is one of the biggest challenges that social researchers will face in the coming years. For this reason, we analyse the relevance that experts have given to family variables in the prevalence of cyberbullying, carrying out a bibliographical review of the relevant research. Among the conclusions reached, it is worth highlighting the need to further explore the importance of the family context as a key variable for the implementation of socio-educational programs focused on the prevention and reduction of cyberbullying.

Keywords: cyberbullying, prevention, intervention, family.
\end{abstract}

\section{Introducción}

Las Tecnologías de la Información y la Comunicación (TIC) han generado grandes cambios en nuestra organización social, afectando a múltiples aspectos de nuestra convivencia y originando también nuevas formas de violencia entre iguales. El cyberbullying constituye un fenómeno social en crecimiento con múltiples efectos a nivel emocional, psicosocial y académico. No olvidemos que, mayoritariamente, las víctimas, observadores y agresores son aún menores de edad.

Sin lugar a dudas, la prevención del cyberbullying constituye uno de los mayores desafíos que deberemos afrontar los investigadores sociales en los próximos años, puesto que todavía son muchos los aspectos sobre los que no disponemos de evidencias científicas.
Justamente el trabajo que presentamos se centra en una dimensión hasta el momento menos explorada en los estudios realizados. Nos referimos a la importancia de los progenitores en la prevención y afrontamiento del acoso entre iguales a través de los medios digitales.

Al respecto debemos resaltar que los programas implementados se han centrado en el trabajo con el alumnado, ya que su formación es uno de los aspectos clave para alcanzar la prevención y el afrontamiento del cyberbullying (Garaigordobil y Martínez-Valderrey, 2015).

No obstante, a nuestro parecer, este es un problema que precisa ser comprendido desde una perspectiva más amplia como nos permite la Teoría Ecológica de Bronfenbrenner (1977). Múltiples factores de cada uno de los sistemas que conforman dicha teoría intervienen en el fenómeno como por ejemplo: las normas culturales (macrosistema), la violencia en los medios de comunicación (exosistema), el contexto del profesorado y la comunidad (mesosistema) y, por último, la escuela, los docentes, el alumnado y, por supuesto, los padres y madres (microsistema) (Hong y Espelage, 2012). A indagar sobre el peso que la investigación ha otorgado a las variables familiares dedicaremos las siguientes páginas.

La violencia entre iguales en el entorno virtual: el cyberbullying

Tal y como ha constatado el Instituto Nacional de Estadística (INE, 2016a; 2016b), el acceso a las TIC es, en términos generales, universal en la población española. Los dispositivos digitales están presentes en la vida diaria de los niños y niñas desde edades cada vez más tempranas, lo que ha generado que algunos autores hablen de generación TIC (Bernete, 2010). En todo caso, esta temprana familiarización con los dispositivos tecnológicos no les exime de las posibilidades de sufrir los riesgos que implica su uso inadecuado y/o irresponsable.

En este contexto, hemos sido testigos del surgimiento de una nueva forma de violencia conocida como cyberbullying (Belsey, 2005; Patchin e Hinduja, 2006; Smith et al., 2008; Willard, 2006; Ybarra y Mitchell, 
2004). Hablar de cyberbullying supone asumir cierta controversia terminológica y que se puede ver reflejada en la multitud de etiquetas que lo designan. El término más empleado por la comunidad científica para denominarlo es este término inglés (véase Belsey, 2005; Smith et al., 2008; Willard, 2006) y que, además, evidencia gran concordancia entre su etimología y las características del fenómeno. Esta pluralidad terminológica constata la existencia de diferentes conceptualizaciones que difieren en función de cada autor, si bien convergen en algunos puntos. Una de las definiciones más aceptadas, es la propuesta por Smith et al. (2008), quienes lo describen como un acto agresivo e intencionado, llevado a cabo entre personas o grupos de manera repetida y contante a lo largo del tiempo, mediante formas electrónicas de contacto. Pese a la carencia de una definición unánime, se han consolidado una serie de características que han sido recogidas en la definición anterior, como son la intención de dañar al otro, la repetición y el desequilibrio de poder lo que dificulta que la víctima se defienda por sí misma (Smith, 2014).

El cyberbullying incide en el desarrollo de los niños/as implicados en este tipo de situaciones. Las víctimas padecen cuadros de ansiedad, tienen una baja autoestima, sufren fobia social o síntomas depresivos y dificultades del sueño, afectando además a su rendimiento académico (Manke, 2005; Wolak, Mitchell y Finkelhor, 2006). Hablamos, por tanto, de un fenómeno con claras repercusiones a nivel emocional, psicosocial y académico. En otras palabras, se trata de problema creciente que afecta "a la salud, a la calidad de vida, al bienestar y al correcto desarrollo de la persona" (Garaigordobil, 2011, p. 245).

Puesto que se trata de un problema relativamente reciente, todavía existen numerosos aspectos sobre los que no disponemos de evidencias empíricas suficientes. En este orden de cosas, una de las cuestiones que precisa de mayor indagación es el análisis de la relevancia de la familia en la prevención del cyberbullying.

\section{La prevención y reducción del cyberbullying: la importancia de la familia}

A todas luces es claro que la familia es un agente educativo de primer orden en el sentido de que, además de determinar la configuración de la personalidad de los hijos y garantizar su estabilidad emocional, es la vía principal de cara a la integración plena en la sociedad, pues en ella vamos componiendo nuestra faceta de ser social. Al respecto, hay incluso quien afirma que entre las funciones que cumple la familia en la actualidad, la más significativa, por sus implicaciones para sus miembros, es su capacidad para generar una red de relaciones basada en el afecto y en el apoyo mutuo, susceptibles de asegurar la posterior orientación social de sus miembros y su bienestar futuro (Musitu y Cava, 2001).

A diferencia de otras instituciones con objetivos más concretos y formalizados, en el contexto familiar se lleva a cabo una acción formativa informal y de carácter continuo, con repercusiones en las distintas etapas de la vida. Además, puesto que su influencia comienza incluso antes de nacer y se mantiene a lo largo de la vida de cada uno de sus miembros, otro de los aspectos que la distinguen y la sitúan como principal agente educativo es que las interacciones que se producen en su seno son recíprocas $\mathrm{y}$, prácticamente, constantes, aunque el tipo de influencia entre sus componentes varía con la edad y con las circunstancias personales (Priegue, 2008).

De entre las funciones educativas básicas de la familia deseamos hacer hincapié en dos aspectos que se relacionan directamente con el tema que nos ocupa. En primer lugar, la responsabilidad de los progenitores en la satisfacción de las necesidades de afecto y apoyo requeridas para un desarrollo normal. La familia constituye un punto de referencia psicológico para los niños y niñas que en ella crecen. Es prioritario que exista un clima afectivo y de compromiso al que se sientan vinculados y al que puedan recurrir a lo largo de su vida cuando se encuentren en momentos difíciles, caso de las que pueden tener que ver con ser víctima $u$ observador de situaciones de acoso. $\mathrm{Y}$ en segundo lugar, los progenitores deben crear un clima familiar que favorezca la estimulación necesaria para convertirse en personas competentes, es decir, un ambiente rico en estímulos que los prepare para poder afrontar las demandas y responsabilidades con las que se encontrarán hasta la edad adulta, lo que necesariamente le exige constituirse como un espacio de comunicación, abierto y flexible, donde cada quién pueda expresarse con claridad y con la suficiente confianza como para manifestar sus preocupaciones.

El diálogo debe convertirse en el principal medio de intercambio de ideas, opiniones y pareceres dentro del medio familiar. En nuestro país, Aguilar (2002) ha comprobado que los niños que hacen uso de un diálogo flexible provienen de familias con una estructuración también flexible. Es decir, en los procesos de relación social sus conversaciones pueden ser igualitarias o jerárquicas, dependiendo de los objetivos que se pretendan alcanzar. Acostumbran a ser niños y niñas que participan activamente en los intercambios comunicacionales, en gran parte, gracias a que su ambiente familiar alberga espacios relacionales basados en el diálogo. En el extremo contrario se encuentran aquellos educados en las familias rígidamente estructuradas, donde el diálogo es poco visible y las decisiones dependen de la voluntad de los adultos. Sus principales déficits son la falta de estimulación para la comunicación y la ausencia de protagonismo en la asunción de responsabilidades. Por último, cabe hablar de las familias estructuradas aleatoriamente, en las que las conversaciones se establecen en horizontal o en vertical, indistintamente. En estos casos, no existen criterios de referencia con un mínimo de estabilidad que permitan desarrollar modelos de comunicación apropiados ni se desarrollan suficientes habilidades comunicativas.

Otra de las variables clave es el afecto. Siguiendo a Vázquez (2004), sabemos que los niños parecen prosperar más cuando sienten el afecto de sus padres, cuando estos ejercen la autoridad orientándolos y 
cuando son receptivos a sus necesidades. Desde una perspectiva similar, García Carrasco y García del Dujo (2001) resaltan la evidencia de la necesidad de una expresión afectiva entre adultos y niños/as en la que prime no la cantidad de muestras sino la calidad de las mismas, siendo especialmente relevante una mayor frecuencia del trato presencial. Más allá de las relaciones paterno-filiales, a través de diversos estudios realizados al respecto, se ha constatado que las uniones caracterizadas por discusiones continuas y enfrentamientos y, lo que es más grave, en las que se dan comportamientos violentos, tienen consecuencias negativas en los hijos, tanto a corto como a largo plazo (Arruabarrena y Paúl, 1999).

Así las cosas, lo que analizaremos seguidamente es el peso que la investigación ha otorgado a estos factores en la prevalencia del cyberbullying.

\section{Variables familiares y cyberbullying: evidencias empíricas}

Tal y como ya comentamos, no disponemos de un amplio número de trabajos que se hayan centrado en el estudio las variables familiares que se relacionan con el cyberbullying (Ortega, Buelga y Cava, 2016). No obstante, para alcanzar el objetivo que nos hemos propuesto tomaremos como referencia tanto las principales aportaciones que se han derivado de los estudios centrados en el acoso entre iguales como aquellas que, específicamente, se refieren al cyberbullying. De hecho, tal y como veremos, algunas variables familiares que tradicionalmente se han relacionado con la prevalencia del acoso entre iguales, vuelven a ponerse sobre la mesa cuando hablamos de acoso mediante los medios y dispositivos digitales.

En particular, tras la revisión que hemos llevado a cabo, encontramos que, por lo general, los expertos señalan la importancia de dos dimensiones: el clima familiar y la comunicación familiar. Respecto de la primera dimensión, en primer lugar, debemos señalar que la importancia del clima familiar como factor de gran relevancia en el normal desarrollo de los menores es una cuestión que ha alcanzado amplios niveles de consenso entre la comunidad científica. Musitu, Román y Gracia (1988) ya hacían referencia hace varias décadas a que los resultados de diferentes investigaciones sobre ambiente familiar y logro del alumnado concluían que las medidas del clima familiar están positivamente relacionadas, entre otros factores, con el ajuste psicosocial de los adolescentes. Los estudios realizados en un período temporal más próximo a nuestros días continúan apuntando en la misma dirección al subrayar la relevancia de que en el hogar predominen relaciones interpersonales estables y sostenidas sobre el afecto y el respecto (Robledo y García, 2009), tanto cuando nos referimos a los progenitores entre sí como en lo que tiene que ver con sus hijos e hijas.

Está claro, por tanto, que la calidad de las relaciones familiares constituyen un factor que incide directamente en la competencia y confianza con la que el individuo afronta el período adolescente (García Zabaleta, 2004). En concreto, los expertos apuntan a varias dimensiones que a su vez se relacionan con el clima familiar (Musitu, Buelga, Lila, y Cava, 2001), entre las que destacamos las relacionadas con los conflictos y la calidad de los vínculos familiares. Justamente, el metaanálisis realizado por Lereya, Samara y Wolke (2013) evidencia que la existencia de un clima familiar negativo hace más vulnerables a los jóvenes para ser intimidados por sus iguales. $Y$ es que se ha relacionado la exposición a situaciones de conflicto marital o de conflicto familiar con una mayor predisposición en los hijos e hijas a la hostilidad, conducta antisocial y violencia escolar (Buelga, Iranzo, Cava, \& Torralba, 2015).

En idéntica dirección, Gomes-Franco y Sendín (2014) indican que si las relaciones familiares están deterioradas esto puede provocar que los niños y niñas pasen más tiempo conectados a internet y busquen suplir sus carencias con la interacción con otras personas en la red, lo que como es bien sabido implica numerosos riesgos.

Así pues, podemos afirmar que la cohesión familiar constituye un factor protector del ajuste social durante la adolescencia (Navarro, Ruiz-Oliva, Larrañaga, \& Yubero, 2015), lo que se suele traducir en el desarrollo de relaciones positivas con el grupo de iguales, reduciendo a su vez las posibilidades de sufrir cyberbullying.

Respecto de la otra dimensión que apuntamos, es decir, la comunicación familiar, el análisis que hemos realizado pone de manifiesto su relación directa con la calidad de la vida en el hogar y la percepción que al respecto tienen sus miembros. Es decir, facilita la convivencia familiar e impregna su evolución en las distintas etapas del ciclo vital. Al respecto existe un claro consenso sobre los efectos positivos que se derivan de una comunicación positiva y eficaz entre sus miembros, al tiempo existe acuerdo al afirmar que una comunicación negativa obstruye el desarrollo familiar. En concreto, el estudio realizado por Larrañaga, Yubero, Ovejero y Navarro, (2016) en el que han participado 813 adolescentes españoles con edades comprendidas entre 12 y 18 años constata la mayor posibilidad de ser víctima si la comunicación en la familia es escasa.

Lo cierto es que los problemas de comunicación en el contexto familiar son un factor de riesgo demostrado en las situaciones de bullying (Musitu, Estévez y Emler, 2007). Pues bien, trabajos realizados atendiendo a esta misma variable y relacionándola con las mayores posibilidades de ser víctima de cyberbullying confirman su asociación con el acoso mediante dispositivos digitales y concluyen que mantener una relación comunicativa óptima en la familia es un factor que protege del uso problemático de Internet (Larrañaga, 2016). Varela Garay (2012) va más allá y en su investigación doctoral confirma que, por lo general, los jóvenes que tienen relaciones con sus progenitores caracterizadas por una comunicación abierta y fluida y por utilizar en el seno familiar estrategias de resolución de conflictos basadas en el diálogo y la compresión, no suelen verse afectados por situaciones que implican violencia. 
Tal y como habíamos comentado previamente, uno de los aspectos que diferencian a la familia respecto a otras instituciones formativas, es que los procesos educativos que tienen lugar en su seno no siempre son intencionales, sino que en numerosas ocasiones se realizan de forma inconsciente. Así es que, desde nuestro punto de vista, las dimensiones a las que venimos de hacer referencia se relacionan directamente con los estilos educativos familiares. Por ejemplo, Parra y Oliva (2006) asocian los estilos autoritarios con una mejor adaptación social, mientras que Martínez y García, (2007) sugieren que los hogares indulgentes consiguen los mismos o mejores resultados que los adolescentes educados en familias autoritativas.

Fuera de nuestras fronteras, $y$ en lo que particularmente tiene que ver con la violencia entre iguales, Lereya, Samara y Wolke (2013), tras analizar 70 estudios publicados entre 1970 y 2012 sobre los factores familiares que inciden en el bullying, otorgan a los estilos parentales un papel clave ya que concluyen que pueden actuar como factor de protección o de riesgo. En el referido trabajo, un estilo democrático, con autoridad pero afectivo, aparece como factor de protección, tanto para ser víctima como para ser agresor. Sin embargo, el estilo negligente se reconoce como un fuerte factor de riesgo para ambos roles, así como la carencia de supervisión o de apoyo parental. En cuanto a los predictores específicos de cada rol, también se confirma que la escasa o pobre comunicación con los hijos e hijas, la violencia en el hogar y la inconsistencia en la disciplina están elevadamente vinculados con el rol de agresor, mientras que la sobreprotección se relaciona con la víctima, probablemente debido a que dificulta el desarrollo de habilidades de resolución de conflicto.

Sobre esta misma cuestión otras investigaciones señalan que los jóvenes agresores proceden de hogares donde predominan las prácticas educativas autoritarias y aquellas caracterizadas por la incoherencia (Estévez, Murgui, Moreno, y Musitu, 2007).

No obstante, lo que dice la investigación es que no disponemos de evidencias empíricas que constaten la asociación entre agresores y víctimas de acoso y estilos educativos parentales, si bien es cierto que existe un cierto nivel de consenso al asociar estilos autoritarios y/o inconsistentes con conductas agresivas, y estilos sobreprotectores $\mathrm{y} / \mathrm{o}$ autoritativos a conductas de victimización escolar (Cerezo, Sánchez, Ruiz, y Arense, 2015).

Estos hallazgos se mantienen tanto para bullying como para cyberbullying pero también existe otro predictor específico de este último fenómeno que está vinculado con las familias, y es el tiempo que dedican los jóvenes a utilizar los teléfonos móviles e Internet. Así lo constatan indagaciones realizadas en otros contextos como el asiático (Liu, Fang y Al, 2012), donde más de 200 millones de adolescentes son usuarios de Internet. Pues bien, los resultados obtenidos relacionan el uso de Internet tanto con la disminución de la comunicación familiar como con el uso patológico de la red, lo que añade una nueva variable a tener en cuenta en el diseño y desarrollo de las propuestas educativas que atiendan a la importancia de la familia en la prevención y abordaje del cyberbullying.

\section{Conclusiones}

El recorrido que hemos realizado por los trabajos que han analizado el peso de las variables familiares en la prevalencia del cyberbullying nos ha permitido comprobar, en primer lugar, que esta es una cuestión en la que es preciso profundizar en mayor medida. Ya los estudios centrados en el bullying han enfatizado la relevancia de los factores ligados a los procesos educativos y comunicativos en la familia, al igual que se han contrastado en los trabajos realizados con adolescentes que han delinquido o con conductas desadaptativas. Por ello, no nos sorprende que cuando se trata de abordar el cyberbullying nuevamente debamos tener presente el papel que juegan los progenitores.

En todo caso, y atendiendo específicamente a las variables familiares, hemos podido confirmar que el apoyo y la implicación de los progenitores constituyen factores protectores del cyberbullying. Por ello pensamos que las intervenciones orientadas a mejorar las prácticas educativas familiares repercutirán positivamente en la prevención de este tipo de violencia entre iguales.

Además, teniendo en cuenta que la conveniencia de intervenir desde edades tempranas, creemos que es de gran importancia empezar a trabajar en esta dirección desde Educación Primaria tanto con el alumnado como con las familias $y$, por supuesto también con los profesionales de los centros educativos.

No obstante, previamente, necesitamos emprender un mayor número de investigaciones que se detengan también en otras las variables vinculadas a las familias y las TIC, tales como, el nivel de conocimientos de los progenitores sobre los usos y riesgos que entrañan los dispositivos digitales y los mecanismos de control puestos en marcha en el hogar para gestionar el uso que los hijos e hijas hacen de estos medios.

\section{Referencias}

Aguilar, M.C. (2002): Educación familiar: una propuesta disciplinar y curricular. Málaga: Aljibe.

Arruabarrena, Mã. \& Paúl, J. (1999): Maltrato a los niños en la familia: evaluación y tratamiento. Madrid: Pirámide.

Belsey, B. (2005). Cyberbullying: An emerging Threat to the "always on" generation. http://www.cyberbullying.ca

Bernete, F. (2010). Los usos de las TIC, relaciones sociales y cambios en la socialización de las y los jóvenes. Revista de Estudios de Juventud, 88, 97-114.

Bronfenbrenner, U. (1977). Toward an experimental ecology of human development. American Psychologist, 32, 513-531.

Buelga, S., Iranzo, B., Cava, M.J. \& Torralba, E. (2015). Psychological Profile of Adolescent Cyberbullying Aggressors. International Journal of Social Psychology, 30(2), 382-406. doi: http://dx.doi.org/10.1080/21711976.2015.1016754 
Cerezo, F., Sánchez, C., Ruiz, C. \& Arense. J. J. (2015). Roles en bullying de adolescentes y preadolescentes, y su relación con el clima social y los estilos educativos parentales. Revista de Psicodidáctica, 20(1), 139-155.

Estévez, E., Murgui, S., Moreno, D., y Musitu, G. (2007). Estilos de comunicación familiar, actitud hacia la autoridad institucional y conducta violenta del adolescente en la escuela. Psicothema, 19(1), 108-113.

Garaigordobil, M. (2011). Prevalencia y consecuencias del cyberbullying: una revisión. International Journal of Psychology and Psychological Therapy, 11(2), 233-254.

Garaigordobil, M. y Martínez-Valderrey, V. (2015). Cyberprogram 2.0. Programa de intervención para prevenir y reducir el ciberbullying. Madrid: Ediciones Pirámide.

García Carrasco, J. y García del Dujo, A. (2001): Teoría de la Educación II. Procesos primarios de formación del pensamiento y la acción. Salamanca: Ediciones Universidad de Salamanca.

García Zabaleta, E. (2004). Conductas desadaptativas en los adolescentes de Navarra. El papel de la familia y la escuela. Tesis Doctoral, Departamento de Psicología y Pedagogía, Universidad de Navarra, Pamplona, España.

Gomes-Franco, F. y Sendín, J. C. (2014). Internet como refugio y escudo social: Usos problemáticos de la Red por jóvenes españoles. Comunicar, 43, 45-53.

Hong, J.S. y Espelage, D.L. (2012). A review of research on bullying and peer victimization in school: An ecological system analysis. Aggression and violent behavior, 17(4), 311-322.

Instituto Nacional de Estadística. (2016a). Encuesta sobre Equipamiento y Uso de Tecnologías de Información y Comunicación en los Hogares. http://www.ine.es/dyngs/INEbase/es/operacion.htm?c $=$ estadistica_C\&cid=1254736176741\&menu=ultiDato s\&idp $=125 \overline{47} 35976608$

Instituto Nacional de Estadística. (2016b). Encuesta sobre Sociedad de la Información y la Comunicación en los centros educativos. https://www.educacion.gob.es/educabase/menu.do?ty $\mathrm{pe}=$ pcaxis\&path $=/$ Educacion $/$ Centros $/$ Sice $/ 2014-15-\mathrm{R}$ $\mathrm{D} / \mathrm{Com} \&$ file $=$ pcaxis $\& \mathrm{l}=\mathrm{s} 0$

Larrañaga, E., Yubero, S., Ovejero, A. y Navarro, R. (2016). Loneliness, parent-child communication and cyberbullying victimization among Spanish youths. Computers in Human Behavior, 65, 1-8.

Lereya, S.T., Samara, M., \& Wolke, D. (2013). Parenting Behavior and the Risk of Becoming a Victim and a Bully/victim: A Meta-analysis Study. Child Abuse \& Neglect, 37(12), 1091-1108. doi: http:// -dx.doi.org/10.1016/j.chiabu.2013.03.001

Liu, Q. X., Fang, X. Y. \& Al. (2012). Parent-adolescent Communication, Parental Internet Use and Internet-Specific Norms and Pathological Internet Use among Chinese Adolescents. Computers in Human Behavior, 28, 1269-1275. (DOI: 10.1016/j.chb.2012. 02. 010).
Manke, B. (2005). The impact of cyberbullying. MindOH! Foundation. Recuperado de http://www.mindoh.com/docs/BM_Cyberbullying.pdf

Martínez, I., y García, J. F. (2007). Impact of parenting styles on adolescents' self-esteem and internalization of values in Spain. The Spanish Journal of Psychology, 10(2), 338-348.

Musitu, G. y Cava, M J. (2001): La familia y la educación. Barcelona: Octaedro.

Musitu, G., Estévez, E. y Emler, N. (2007). Adjustment problems in the family and school contexts, attitude towards authority and violent behaviour at school in adolescence. Adolescence, 42, 779-794.

Musitu, G., Román, J.M. y Gracia, E. (1988). Familia y educación. Prácticas educativas de los padres y socialización de los hijos. Barcelona: Labor.

Musitu, G.; Buelga, S.; Lila, M. y Cava, M. J. (2001). Familia y adolescencia. Madrid: Síntesis.

Navarro, R., Ruiz-Oliva, R., Larrañaga, E., \& Yubero, S. (2015). The Impact of Cyberbullying and Social Bullying on Optimism, Global and School-related Happiness and Life Satisfaction among 10-12-year-old Schoolchildren. Applied Research in Quality of Life, 10(1), 15-36. doi: http://dx.doi.org/ 10.1007/s11482-013-929-2-0

Ortega, J., Buelga, S., y Cava, M. J. (2016) Influencia del clima escolar y familiar en adolescentes, víctimas de ciberacoso. Comunicar, 46, 57-65.

Parra, A., y Oliva, A. (2006). Un análisis longitudinal sobre las dimensiones relevantes del estilo parental durante la adolescencia. Infancia y Aprendizaje, 29(4), 453-470.

Patchin, J. W., e Hinduja, S. (2006). Bullies move beyond the Schoolyard: A preliminary look at cyberbullying. Youth violence and juvenile justice, 4(2), 148-169.

Priegue, D. (2008). Familia, educación e inmigración. Un programa de intervención pedagógica. Tesis Doctoral, Departamento de Teoría de la Educación, Historia de la Educación y Pedagogía Social, Universidade de Santiago de Compostela, Santiago de Compostela, España. https://minerva.usc.es/

Robledo, P. y García, J (2009) El entorno familiar y su influencia en el rendimiento escolar de los alumnos con dificultades de aprendizaje: revisión de estudios empíricos. Aula Abierta, 17(1), 117-128.

Smith, P. K., Mahdavi, J., Carvalho, M., Fisher, S., Russell, S., y Tippett, N. (2008). Cyberbullying: Its nature and impact in secondary school pupils. Journal of Child Psychology and Psychiatry, 49, 376-385.

Smith, P.K. (2014). Understanding School Bullying. London: Sage Publications.

Varela Garay, R. M. (2012). Violencia, Victimización y Cyberbullying en adolescentes escolarizados/as: una perspectiva desde el Trabajo Social. Tesis Doctoral, Departamento de Trabajo Social y Servicios Sociales, Universidad Pablo Olavide, Sevilla, España. http://www.uv.es/lisis/rosavarela/tesis-rosa-varela.pdf

Vázquez, G. (2004): Familia, educación y desarrollo cognitivo. En M.A. Santos Rego, M.A. \& J.M. Touriñan López, J.M. (Eds.), Familia educación y sociedad civil (pp. 29-87). Santiago de Compostela: 
Servizo de Publicacións e Intercambio Científico da Universidade de Santiago de Compostela.

Willard, N. (2006). Cyberbullying and Cyberthreats. Effectively managing internet use risks in schools. http://new.csriu.org/cyberbully.pdf

Wolak J., Mitchell K. \& Finkelhor D. (2006). Online victimization of youth: Five years later. http://www.unh.edu/ccrc/pdf/CV138.pdf

Ybarra, M. L. \& Mitchell, K. J. (2004). Online aggressor/targets, aggressors, and targets: A comparison of associated youth characteristics. Journal of Child Psychology and Psychiatry, 45, 1308-1316. 